\title{
Rapid detection of urinary tract infections caused by Proteus spp. using PNA-FISH
}

\author{
C. Almeida $\cdot$ N. F. Azevedo $\cdot$ J. C. Bento $\cdot$ N. Cerca . \\ H. Ramos • M. J. Vieira • C. W. Keevil
}

Received: 7 May 2012 / Accepted: 18 December 2012 /Published online: 4 January 2013

(C) Springer-Verlag Berlin Heidelberg 2013

\begin{abstract}
We developed a fluorescence in situ hybridization (FISH) method for the rapid detection of Proteus spp. in urine, using a novel peptide nucleic acid (PNA) probe. Testing on 137 urine samples from patients with urinary tract infections has shown specificity and sensitivity values of $98 \%(95 \%$ CI, 93.2-99.7) and $100 \%$ (95\% CI, 80,8-100), respectively, when compared with CHROMagar Orientation medium. Results indicate that PNA-FISH is a reliable alternative to traditional culture methods and can reduce the diagnosis time to approximately $2 \mathrm{~h}$.
\end{abstract}

Electronic supplementary material The online version of this article (doi:10.1007/s10096-012-1808-2) contains supplementary material, which is available to authorized users.

C. Almeida $\cdot$ J. C. Bento $\cdot$ N. Cerca $\cdot$ M. J. Vieira

IBB - Institute for Biotechnology and Bioengineering,

Centre of Biological Engineering, Universidade do Minho,

Campus de Gualtar,

4710-057 Braga, Portugal

\section{Almeida $\cdot$ C. W. Keevil}

Environmental Healthcare Unit, School of Biological Sciences,

University of Southampton, Bassett Crescent East,

SO16 7PX Southampton, UK

C. Almeida $\cdot$ N. F. Azevedo

LEPAE, Department of Chemical Engineering,

Faculty of Engineering, University of Porto, rua Dr. Roberto Frias, 4200-465 Porto, Portugal

H. Ramos

Serviço de Microbiologia, Centro Hospitalar do Porto,

Hospital Geral de Santo António, 4000 Porto, Portugal

C. Almeida $(\bowtie)$

Centro de Engenharia Biólogica, Universidade do Minho,

4710-057 Braga, Portugal

e-mail: carinaalmeida@deb.uminho.pt

\section{Introduction}

The genus Proteus is in the top five causes of urinary tract infections (UTIs) [1, 2] and is related to the emergence of complicated UTI (10-15\% of the cases), mainly for immuno-compromised patients [3]. Proteus-associated UTIs can be difficult to treat and are usually associated with bladder and kidney stone formation, that can lead to the obstruction of the urinary tract and catheters [3, 4]. The three species of Proteus described as being associated with UTIs are Proteus mirabilis, Proteus vulgaris and Proteus penneri. P. mirabilis is considered to be the third most common cause of complicated UTI (responsible for $12 \%$ of infections) and the second most common cause of catheter-associated UTI's (CAUTI's) in long-term catheterized patients (15\%) [4].

In terms of UTI diagnosis, current techniques require 24$48 \mathrm{~h}$ to identify pathogenic species in urine $[5,6]$. Chromogenic agars, such as CHROMagar Orientation and Candida and MacConkey agar, have been developed to facilitate and accelerate species recognition directly on primary media, and have been widely adopted [7, 8]. However, studies have shown that results obtained using the different chromogenic media are not consistent, because microorganisms are not always detected on media, and the colon counts and species recognized differed $[6,9]$. In the absence of expeditious microbiological diagnosis, clinicians must initiate empirical antimicrobial treatment without supportive laboratory data, which has led to the emergence of resistant pathogens. Thus, a rapid molecular approach would be beneficial.

Fluorescence in situ hybridization (FISH) is a molecular method used to identify and quantify microbial populations [10]. The combination of this method with peptide nucleic acid (PNA) probes has shown to have many advantages compared to conventional DNA-FISH [11]. PNA-FISH 
has been applied to the detection of several clinical relevant microorganisms on a broad range of samples (for a review see [12]). More recently, some studies have shown that its implementation as a routine method in clinical laboratories allows for a rational use of medicines, and reduces hospitalization times and mortality $[13,14]$.

In this work we developed and validated a new PNAFISH method for clinical detection of Proteus in urine samples from patients with UTIs.

\section{Material and methods}

Culture maintenance

The bacterial strains used in this study are listed in Table 1. Streptococcus and Campylobacter strains were maintained on Columbia agar (Oxoid) supplemented with $5 \%$ (vol/ $/ \mathrm{vol}$ ) defibrinated horse blood (Probiológica) and incubated at $37^{\circ} \mathrm{C}$, $10 \% \mathrm{CO}_{2}$ and $5 \% \mathrm{O}_{2}$ in a $\mathrm{CO}_{2}$ incubator (HERAcell 150, Waltham). Single colonies were streaked onto fresh plates every 2-3 days. All remaining bacterial species were maintained on Tryptic Soy Agar (Sigma) at $37{ }^{\circ} \mathrm{C}$ and restreaked every $24 \mathrm{~h}$.

PNA probe design and theoretical evaluation

To identify potentially useful oligonucleotides for use as probe, the Primrose program was used coupled with the 16S rRNA databases of the Ribosomal Database Project II (RDPII) (http://rdp.cme.msu.edu/html) [15, 16]. Criteria for the selection of the PNA probe included: high number of Proteus detected, low number of non-targets detected, no self-complementary structures, and lower number of nontarget sequences with one mismatch. The selected sequence was synthesized (Panagene, South Korea) and the N-terminus attached to Alexa fluor 488 via a double AEEA linker. The theoretical specificity and sensitivity of the probe was evaluated with the updated databases available at RDPII (RDP Release 10, Update 28, January 2012) and NCBI.

Specificity was calculated as $\mathrm{nPs} /(\mathrm{TnP}) \mathrm{x} 100$, where $\mathrm{nPs}$ stands for the number of non-Proteus strains that did not react with the probe and $\mathrm{TnP}$ for total of non-Proteus strains examined. Sensitivity was calculated as Ps/(TPs)x100, where Ps stands for the number of Proteus strains detected by the probe and TPs for the total number of Proteus strains in the databases.

Hybridization procedure

The hybridization procedures were performed as previously reported [17]. For the protocol optimization the hybridization was repeated but hybridization and washing temperatures (57 to $65^{\circ} \mathrm{C}$ ) as well as the hybridization time (45 and $90 \mathrm{~min}$ ) were changed.

After the hybridization, samples were allowed to airdry, mounted with one drop of non-fluorescent immersion oil (Merck) and covered with coverslips. Samples were visualized in an epifluorescence microscope (Olympus BX51) with an FITC filter. A negative control was performed simultaneously, with hybridization solution without probe.

\section{Proteus probe specificity test}

The specificity of the probe was tested using 22 Proteus strains of different species and 52 other strains. These latter strains included 32 taxonomically related strains of the same family, and 20 strains of different orders, classes or phyla.

Detection limit in artificial urine

To evaluate the detection limit, artificial urine samples, prepared as reported before [18], were inoculated with $P$. vulgaris ATCC29905 and P. mirabilis ATCC 21100 (grown overnight in artificial urine at $37{ }^{\circ} \mathrm{C}, 120 \mathrm{rpm}$ ) with concentrations ranging from $1 \times 10^{2}$ to $1 \times 10^{7} \mathrm{CFU} /$ $\mathrm{mL}$. As the detection limit reported for PNA-FISH is $2 \times 10^{5}$ cells $/ \mathrm{mL}$ [17], $20 \mathrm{~mL}$ samples (which were 20fold concentrated by centrifugation at $10,000 \mathrm{~g}$ for $5 \mathrm{~min}$ ) were used before starting the hybridization procedure. This was performed in suspension or on glass slides as described above.

To quantify the number of cells lost during the PNAFISH method, samples were taken during the different procedure steps (inoculum in water, paraformaldehyde, ethanol, washing solution 1 and washing solution 2), stained with DAPI and counted under the microscope.

Quantification of cells by DAPI or PNA-FISH was obtained by counting a total of 15 fields with an area of $0.0158 \mathrm{~mm}^{2}$. The average was used to calculate total cells per $\mathrm{mL}$ of sample. These experiments were performed three times.

Detection in urine samples

To evaluate method performance on real samples, 78 urine samples from outpatients with symptoms of UTI, were collected in sterile flasks at Hospital de S. João (Porto, Portugal) and Laboratório de Análises Clínicas S. Lázaro (Braga, Portugal) and simultaneously analyzed by PNA-FISH and CHROMagar Orientation medium. For PNA-FISH analyses, hybridization was performed as described above, but for turbid urine samples only $1 \mathrm{ml}$ was pelleted by centrifugation. 
Table 1 Results of the Proteus probe (ProPNA190) specificity and sensitivity test
${ }^{\mathrm{a}}$ Type strains
${ }^{\mathrm{b}}$ Isolates
Microoganisms

PNA

FISH

outcome

Proteus mirabilis ATCC $29906^{\mathrm{a}}$, ATCC $14153^{\mathrm{a}}$, ATCC $21100^{\mathrm{a}}, 414^{\mathrm{b}}, 417^{\mathrm{b}}, 933^{\mathrm{b}}, \mathrm{M}^{\mathrm{b}}{ }^{\mathrm{b}}$, PL050596 ${ }^{\mathrm{b}}, \mathrm{PL} 050835^{\mathrm{b}}, \mathrm{PL} 49417^{\mathrm{b}}, \mathrm{PL} 49905^{\mathrm{b}}$ and PL50359 ${ }^{\mathrm{b}}$

Proteus vulgaris ATCC $6380^{\mathrm{a}}$, ATCC $6896^{\mathrm{a}}$, ATCC $9484^{\mathrm{a}}$, ATCC $29905^{\mathrm{a}}, \mathrm{M} 12^{\mathrm{b}}, 404^{\mathrm{b}}$ and $43^{\mathrm{b}}+$

Proteus penneri ATCC $33519^{\mathrm{a}}$, CDC2518-74 ${ }^{\mathrm{a}}$

Proteus hauseri ATCC $13315^{\mathrm{a}}$

Cronobacter sakazakii ATCC $29544^{\mathrm{a}}$

Cronobacer malonaticus ${ }^{\mathrm{b}}$

Enterobacter aerogenes CECT $684^{\mathrm{a}}$

Enterobacter amnigenus CECT $4078^{\text {a }}$

Enterobacter asburiae ${ }^{\mathrm{b}}$

Enterobacter cloacae ${ }^{\mathrm{b}}$

Enterobacter helveticus ${ }^{\mathrm{b}}$

Enterobacter hormaechei ${ }^{\mathrm{b}}$

Escherichia coli $\mathrm{K} 12^{\mathrm{a}}$, ATCC $25922^{\mathrm{a}}$, $\mathrm{N} 5^{\mathrm{b}}$ and N9 ${ }^{\mathrm{b}}$

Escherichia hermannii ATCC $33650^{\mathrm{a}}$

Escherichia vulneris ATCC $29943^{\mathrm{a}}$

Klebsiella oxytoca ATCC $13182^{\mathrm{a}}$

Klebsiella pneumoniae ATCC $11296^{\mathrm{a}}$

Citrobacter freundii ${ }^{\mathrm{b}}$

Serratia plymuthica $\mathrm{F}^{\mathrm{b}}$

Morganella morganii CDC4195-69

Salmonella bongori SGSC $3100^{\mathrm{a}}$, SGSC $3103^{\mathrm{a}}$

Salmonella enterica subp. enterica serovar Enteritidis SGSC $2474^{\text {a }}$

Salmonella enterica subp. enterica serovar Newport SGSC $2493^{\mathrm{a}}$

Salmonella enterica subp. enterica serovar Panama SGSC $2497^{\mathrm{a}}$

Salmonella enterica subp. enterica serovar Paratyphi C SGSC $2506^{\mathrm{a}}$

Salmonella enterica subsp. enterica serovar Indiana SGSC $2482^{\mathrm{a}}$

Salmonella enterica subp. enterica serovar Dublin SGSC 2470a

Shigella boydii ATCC $9207^{\mathrm{a}}$

Shigella dysenteriae ATCC $11835^{\mathrm{a}}$

Shigella sonnei ATCC 25931 ${ }^{\mathrm{a}}$

Yersinia kritensenii PL115185 $5^{\mathrm{b}}$

Yersinia enterocolitica subsp. enterocolitica PL129178

Pseudomonas aeuroginosa ATCC $10145^{\text {a }}$

Pseudomonas fluorescens ATCC $13525^{\mathrm{a}}$

Campylobacter coli ${ }^{\mathrm{b}}$

Campylobacter jejuni ${ }^{\mathrm{b}}$

Staphylococcus aureus subsp. aureus ATCC $13565^{\mathrm{a}}$, ATCC $12600^{\mathrm{a}}$, ATCC $6538^{\mathrm{a}}$ and $239^{\mathrm{b}}$

Staphylococcus sciuri ${ }^{\mathrm{b}}$

Staphylococcus epidermidis ATCC 35983 ${ }^{\mathrm{a}}$; ATCC 35984 ${ }^{\mathrm{a}}$; ATCC $1798^{\mathrm{a}}$; ATCC $14990^{\mathrm{a}}$

Streptococcus mutans $\mathrm{UC}^{\mathrm{b}}, \mathrm{UP}^{\mathrm{b}}$

Enterococcus faecalis ATCC $27285^{\mathrm{a}}$

Enterococcus faecium ATCC $19434^{\mathrm{a}}$

Listeria monocytogenes CECT $4031 \mathrm{~T}^{\mathrm{a}}, 747^{\mathrm{b}}, 924^{\mathrm{b}}$
For culture analyses, a $10-\mu \mathrm{L}$ volume of each sample was spread on CHROMagar Orientation and incubated for 24 or
$48 \mathrm{~h}$ at $37{ }^{\circ} \mathrm{C}$. Only samples with Proteus concentrations higher than $1 \times 10^{4} \mathrm{CFU} / \mathrm{ml}$ were considered positive for UTI. 


\section{Results}

Probe design

The selection of oligonucleotides was based on the $16 \mathrm{~S}$ rRNA comparison of all Proteus spp. strains in the RDP II database. Several potentially useful oligonucleotides were identified using the PRIMROSE software. From the possible probes identified, and based on criteria described in "Material and Methods", we selected the following PNA oligomer sequence: 5'-GCCCCTGCTTTGGTC- 3'. This probe hybridizes between positions 190 and 204 of the $P$. mirabilis strain HI4320 16S rRNA (accession number: NC_010554.1) and was thus designated ProPNA190.

The search showed that the ProPNA190 detected 135 of 140 Proteus sequences present in the database (last accession, March 2012), and thus a sensitivity of $96.4 \%$ was obtained. The five non-detected Proteus sequences were three $P$. mirabilis (accession number: S000806301, S002305608 and S002409155), one P. vulgaris (S000414235) and one Proteus spp. (S002950968). To confirm this result the five sequences of rDNA were aligned with the probe reverse-complement. We observed that sequence S002950968 was incomplete and did not include the region where the probe hybridizes. Sequences S000414235, S000806302, S002305608, and S002409155 present one mismatch of base pairs compared to ProPNA190 (Supplemental material, Fig. S1). For the $P$. mirabilis S000806301 strain the mismatch corresponds to an $\mathrm{R}(\mathrm{A} / \mathrm{G})$ in the rRNA sequence, meaning that this strain might also be detectable with the probe. From the 495940 16S rRNA sequences analyzed, ProPNA190 also detected four non-Proteus sequences: one Orientia tsutsugamushi (S000266975), two Idiomarina sediminum (S000842774 and S002446946) and one Pseudidiomarina sp. S001098108 (Pseudidiomarina spp. inclusion in Idiomarina genus have been recently proposed [19]). However, none of these non-targets is relevant to the diagnosis of urinary infection [20, 21]. As we analyzed 495,851 non-Proteus strains and only two were detected by the probe, a theoretical specificity of $99.99 \%$ was obtained.

Probe specificity and sensitivity testing

The hybridization conditions for ProPNA190 were optimized and a strong signal-to-noise ratio was obtained from $55^{\circ} \mathrm{C}$ to $65{ }^{\circ} \mathrm{C}$ for $45 \mathrm{~min}$ of hybridization. However, to achieve an appropriate specificity, $65{ }^{\circ} \mathrm{C}$ was used for all subsequent tests. After optimization, the specificity and sensitivity of the PNA probe were tested on 22 representative Proteus strains and another 52 strains, including common urinary pathogens, such as E. coli, E. faecalis, K. pneumonia and Staphylococcus [22].

As shown in Table 1, all Proteus strains were detected, whereas no hybridization was observed for the other species used. Therefore, experimental specificity and sensitivity were both $100 \%$ (sensitivity, $95 \%$ CI, 81.5-100, and specificity, $95 \%$ CI, 91.4-100).

Detection limit in artificial urine

To achieve the desired sensitivity, and considering the reported detection limit for PNA-FISH of $2 \times 10^{5}$ cells $/ \mathrm{mL}$ [17], samples were 20 -fold concentrated by centrifugation before the hybridization procedure.

As the PNA-FISH method also involves centrifugation steps, the number of cells lost during the procedure was determined to assure that it did not interfere with the PNA-FISH result. We observed that the percentage of cells lost during the procedure was 7-8 \% (Table S1), and the total cells lost from the initial step till the end of the PNA-FISH method was $26.39 \%( \pm 7.46)$. Therefore, and considering that we are measuring total cells that are even higher than CFU counts (for Proteus in artificial urine, the number of cells detected by cultivability was $85.75 \pm$ $3.65 \%$ ), we were assured that this loss does not interfere with the outcome. In Fig. 1 we can observe the results obtained for each bacterial concentration. This procedure was able to detect $1 \times 10^{4} \mathrm{CFU} / \mathrm{mL}$ (corresponding to $\sim 10$ cells/microcopy field; Supplemental material Table S2), while concentrations lower than this were not detected. However, if a concentration of $1 \times$ $10^{5} \mathrm{CFU} / \mathrm{mL}$ is desired as indicative of infection, a maximum initial urine volume of $2 \mathrm{~mL}$ should be used.

\section{Detection of Proteus in clinical urine specimens}

To evaluate method performance on real samples, urine from outpatients with symptoms of UTI were collected and simultaneously analyzed by PNA-FISH and CHROMagar Orientation medium. For CHROMagar Orientation, only samples with Proteus concentrations higher than $1 \times 10^{4} \mathrm{CFU} / \mathrm{ml}$ were considered positive for UTI. For PNA-FISH a positive outcome was always considered indicative of UTI (Fig. 2 shows a positive result for Proteus presence in a real urine sample). As shown in Table 2, from the 137 samples analyzed, only 23 were positive for Proteus presence using the CHROMagar Orientation medium. Using this PNA-FISH method, no false negative results were observed, but two false positive results were obtained. As such, the observed value of sensitivity was $100 \%$, with a confidence interval (CI) of $95 \%$, from 80.8 to $100 \%$, and for specificity a value of $98 \%$ was determined, with a CI from 93.2 to $99.7 \%$. Results were quite consistent between the two techniques, with the main difference being the time required to achieve the final result.

\section{Discussion}

The Proteus genus includes five species, $P$. vulgaris, $P$. mirabilis, $P$. penneri, $P$. hauseri and $P$. myxofaciens, which 
Fig. 1 Epifluorescence detection of $P$. vulgaris ATCC 29905 using the ProPNA190 probe in artificial urine with $1 \times$ $10^{7}$ (a), $1 \times 10^{6}$ (b), $1 \times 10^{5}(\mathbf{c})$ and $1 \times 10^{4}$ (d) CFU per $\mathrm{mL}$ of urine
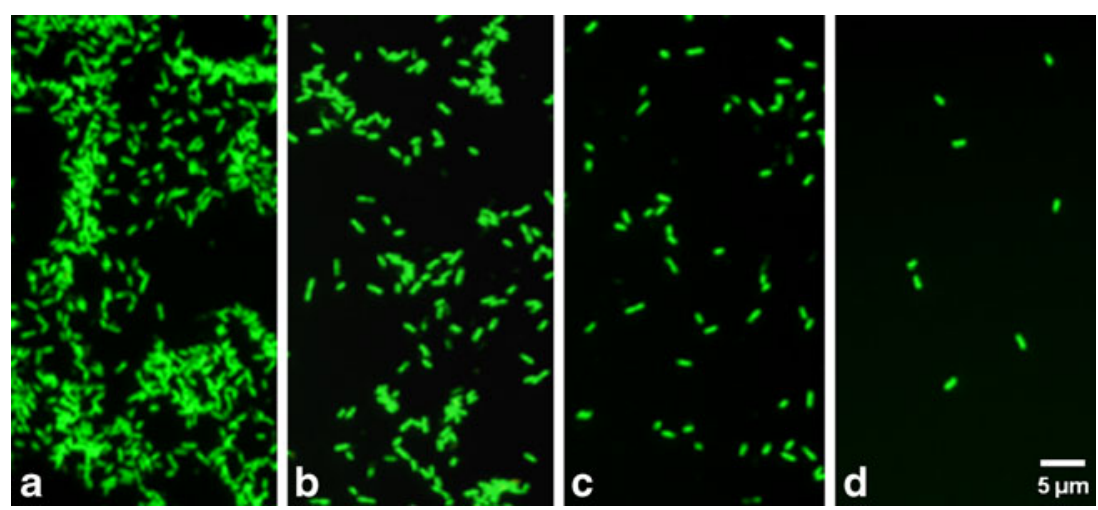

exist in soil, polluted water, and intestines of humans and a wide variety of animals [23, 24]. For the probe specificity test, we included four Proteus species: P. vulgaris, P. mirabilis, $P$. penneri and $P$. hauseri, though only $P$. vulgaris, $P$. mirabilis and $P$. penneri species are reported as causing UTIs [22, 23]. $P$. hauseri is not usually reported as a cause of UTI's, because it is considered the genomospecies 3 of $P$. vulgaris species [25]. Additionally, 32 taxonomically related strains of the same family (Shigella, Klebsiella, Citrobacter, Pantoea, Yersinia, Enterobacter, Escherichia and Serratia, Morganella) and 20 strains of different orders (Pseudomonas), classes (Campylobacter) or phyla (Listeria, Streptococcus, Enterococcus and Staphylococcus) were also tested, and no cross-hybridization was observed for the non-Proteus strains tested.
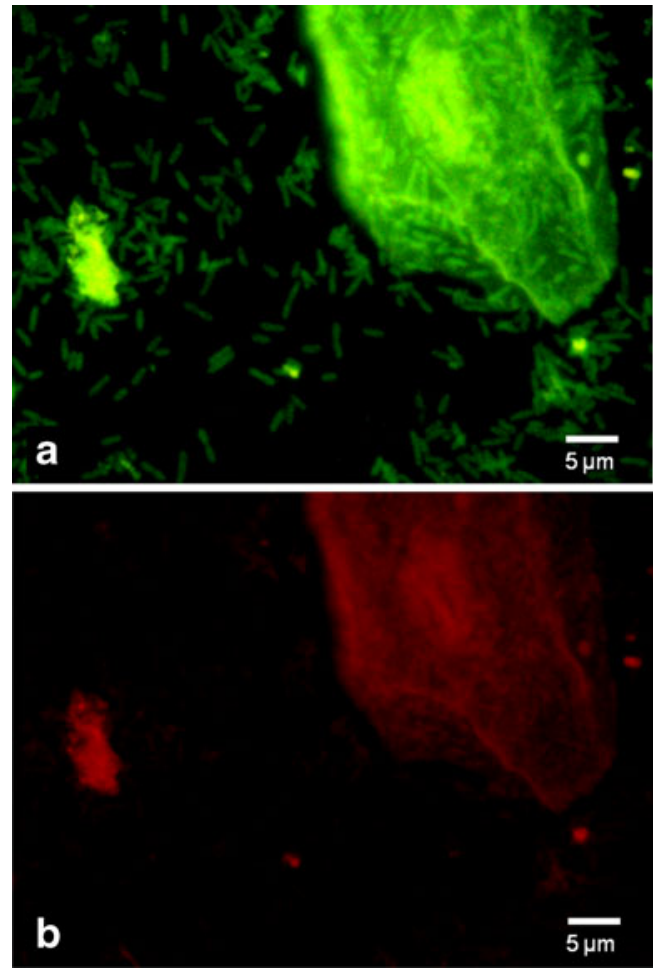

Fig. 2 Detection of Proteus in a real urine sample using the ProPNA190 probe. a Image at the green filter capable of detecting the Alexa 488 (BP 470-490, FT 500, LP 516 for LmPNA1253). b Image at the red filter (BP 530-550, FT 570, LP 591)
After the probe testing the PNA FISH was adapted for the detection of UTIs caused by Proteus spp. in urine samples. Currently, urinalysis and the detection of nitrites in urine are the most used UTI diagnosis assays [26]. However, they are not able to identify the pathogen. The only assay capable of this and of establishing a definitive diagnosis of UTI is urine culture, a method pioneered by Koch and Petri in the 1880s, and refined during the last several decades. The latest urine culture technology allows the identification of various organisms in a single test since various pathogens grown on a chromogenic medium produce a color change that is species specific. However these tests usually require $24-48 \mathrm{~h}$, meaning that clinicians need to start the empirical treatment before having urine culture result $[5,6]$. An urine culture of at least $10^{5} \mathrm{CFU} / \mathrm{mL}$ of any single type of bacterium has traditionally been believed to provide conclusive evidence of UTI [26, 27]; although, for catheterized individuals this value can be lower $\left(\sim 10^{4} \mathrm{CFU} / \mathrm{ml}\right)$ [28]. Therefore, as Proteus is associated with the infection of catheterized patients [3, 4], a threshold of $1 \times$ $10^{4} \mathrm{CFU} / \mathrm{mL}$ was defined. Using the PNA-FISH method described here, we were able to detect as low as $1 \times 10^{4}$ $\mathrm{CFU} / \mathrm{mL}$ in approximately $2 \mathrm{~h}$, with similar accuracy (sensitivity of $100 \%$ and specificity of $98 \%$ ) to the standard culture method (Chromagar Orientation).

Several researchers have been working on the development of new techniques to reduce the time and cost of urine culture processing. However, while some methods, such as PCR, are unable to distinguish between clinically significant or nonsignificant bacteriuria, others like the use of chromogenic

Table 2 PNA-FISH and CHROMagar results for Proteus presence in real urine samples. The presence of Proteus in CHROMagar was considered only for concentrations higher than $1 \times 10^{4} \mathrm{CFU} / \mathrm{ml}$ of urine

\begin{tabular}{lllll}
\hline Measurement & & \multicolumn{3}{l}{ CHROMagar } \\
\cline { 3 - 5 } & & Absence & Presence & Total \\
\hline PNA-FISH & Positive & 2 & 21 & 23 \\
& Negative & 114 & 0 & 114 \\
& Total & 116 & 21 & 137 \\
\hline
\end{tabular}


media are limited by the slow growth, or inability to grow, of some microorganisms $[6,9,29]$.

The protocol presented in this work has been demonstrated to be a reliable alternative to the currently used culture-based techniques. It also presented an appropriate level of sensitivity for detection of clinically relevant concentrations of bacteria in urine. The method is also likely to be adapted for a multiplex detection of other common urinary pathogens, as previously performed for foodborne pathogens [30].

Acknowledgments We would like to thank Cristina Silva for providing us the urine samples. This work was supported by Fundação para a Ciência e Tecnologia (Postdoctoral Fellowship SFRH/BPD/ $74480 / 2010$ and project PIC/IC/82815/2007) and by the European Commission's Framework Programme (FP7: “COST-Action TD1004: Theragnostics for imaging and therapy").

Conflict of interest The authors declare that they have no conflict of interest.

\section{References}

1. Gordon KA, Jones RN (2003) Susceptibility patterns of orally administered antimicrobials among urinary tract infection pathogens from hospitalized patients in North America: comparison report to Europe and Latin America. Results from the sentry antimicrobial surveillance program (2000). Diagn Microbiol Infect Dis 45:295-301

2. Farrell DJ, Morrissey I, De Rubeis D, Robbins M, Felmingham D (2003) A UK multicentre study of the antimicrobial susceptibility of bacterial pathogens causing urinary tract infection. J Infect 46:94-100

3. Cohen-Nahum K, Saidel-Odes L, Riesenberg K, Schlaeffer F, Borer A (2010) Urinary tract infections caused by multi-drug resistant proteus mirabilis: Risk factors and clinical outcomes. Infection 38:41-46

4. Jacobsen SM, Stickler DJ, Mobley HL, Shirtliff ME (2008) Complicated catheter-associated urinary tract infections due to escherichia coli and proteus mirabilis. Clin Microbiol Rev 21:26-59

5. Pavlou AK, Magan N, McNulty C et al (2002) Use of an electronic nose system for diagnoses of urinary tract infections. Biosens Bioelectron 17:893-899

6. D'Souza HA, Campbell M, Baron EJ (2004) Practical bench comparison of bbl chromagar orientation and standard two-plate media for urine cultures. J Clin Microbiol 42:60-64

7. Chaux C, Crepy M, Xueref S, Roure C, Gille Y, Freydiere AM (2002) Comparison of three chromogenic agar plates for isolation and identification of urinary tract pathogens. Clin Microbiol Infect 8:641-645

8. Scarparo C, Piccoli P, Ricordi P, Scagnelli M (2002) Comparative evaluation of two commercial chromogenic media for detection and presumptive identification of urinary tract pathogens. Eur $\mathbf{J}$ Clin Microbiol Infect Dis 21:283-289

9. Aspevall O, Osterman B, Dittmer R, Sten L, Lindback E, Forsum U (2002) Performance of four chromogenic urine culture media after one or two days of incubation compared with reference media. J Clin Microbiol 40:1500-1503

10. Amann R, Fuchs BM (2008) Single-cell identification in microbial communities by improved fluorescence in situ hybridization techniques. Nat Rev Microbiol 6:339-348

11. Stender H (2003) Pna fish: an intelligent stain for rapid diagnosis of infectious diseases. Expert Rev Mol Diagn 3:649-655

12. Cerqueira L, Azevedo NF, Almeida C, Jardim T, Keevil CW, Vieira MJ (2008) DNA mimics for the rapid identification of microorganisms by fluorescence in situ hybridization (fish). Int J Mol Sci 9:1944-1960
13. Forrest GN (2007) Pna fish: present and future impact on patient management. Expert Rev Mol Diagn 7:231-236

14. Forrest GN, Roghmann MC, Toombs LS et al (2008) Peptide nucleic acid fluorescent in situ hybridization for hospital-acquired enterococcal bacteremia: delivering earlier effective antimicrobial therapy. Antimicrob Agents Chemother 52:3558-3563

15. Ashelford KE, Weightman AJ, Fry JC (2002) Primrose: a computer program for generating and estimating the phylogenetic range of 16s rRNA oligonucleotide probes and primers in conjunction with the RDP-II database. Nucleic Acids Res 30:3481-3489

16. Cole JR, Chai B, Farris RJ et al (2005) The ribosomal database project (RDP-II): sequences and tools for high-throughput rrna analysis. Nucleic Acids Res 33:D294-D296

17. Almeida C, Azevedo NF, Iversen C, Fanning S, Keevil CW, Vieira MJ (2009) Development and application of a novel peptide nucleic acid probe for the specific detection of Cronobacter genomospecies (Enterobacter sakazakii) in powdered infant formula. Appl Environ Microbiol 75:2925-2930

18. Brooks T, Keevil CW (1997) A simple artificial urine for the growth of urinary pathogens. Lett Appl Microbiol 24:203-206

19. Taborda M, Antunes A, Tiago I, Verissimo A, Nobre MF, da Costa MS (2009) Description of Idiomarina insulisalsae sp. nov., isolated from the soil of a sea salt evaporation pond, proposal to transfer the species of the genus Pseudidiomarina to the genus Idiomarina and emended description of the genus Idiomarina. Syst Appl Microbiol 32:371-378

20. Jean WD, Leu TY, Lee CY, Chu TJ, Lin SY, Shieh WY (2009) Pseudidiomarina marina sp. nov. and Pseudidiomarina tainanensis sp. nov. and reclassification of Idiomarina homiensis and Idiomarina salinarum as Pseudidiomarina homiensis comb. nov. and Pseudidiomarina salinarum comb. nov., respectively. Int J Syst Evol Microbiol 59:53-59

21. Tantibhedhyangkul W, Prachason T, Waywa D, et al (2011) Orientia tsutsugamushi stimulates an original gene expression program in monocytes: relationship with gene expression in patients with scrub typhus. Plos Negl Trop Dis 5:e1028

22. Ronald A (2002) The etiology of urinary tract infection: traditional and emerging pathogens. Am J Med 113:14s-19s

23. Cao B, Wang M, Liu L et al (2009) 16s-23s rdna internal transcribed spacer regions in four proteus species. J Microbiol Methods 77:109-118

24. O'Hara CM, Brenner FW, Miller JM (2000) Classification, identification, and clinical significance of proteus, providencia, and morganella. Clin Microbiol Rev 13:534-546

25. O'Hara CM, Brenner FW, Steigerwalt AG et al (2000) Classification of Proteus vulgaris biogroup 3 with recognition of Proteus hauseri sp. nov., nom. rev. and unnamed Proteus genomospecies 4, 5 and 6. Int J Syst Evol Microbiol 50:1869-1875

26. Nickel JC (2005) Management of urinary tract infections: historical perspective and current strategies: part 1 - before antibiotics. J Urol 173:21-26

27. American Academy of Pediatrics (1999) Practice parameter: the diagnosis, treatment, and evaluation of the initial urinary tract infection in febrile infants and young children. American Academy of Pediatrics. Committee on quality improvement. Subcommittee on urinary tract infection. Pediatrics 103:843-852

28. Hoberman A, Wald ER, Reynolds EA, Penchansky L, Charron M (1994) Pyuria and bacteriuria in urine specimens obtained by catheter from young children with fever. J Pediatr 124:513-519

29. Liao JC, Mastali M, Gau V et al (2006) Use of electrochemical DNA biosensors for rapid molecular identification of uropathogens in clinical urine specimens. J Clin Microbiol 44:561-570

30. Almeida C, Azevedo NF, Santos S, Keevil CW, Vieira MJ (2011) Discriminating multi-species populations in biofilms with peptide nucleic acid fluorescence in situ hybridization (PNA FISH). Plos One 6:e14786 